\title{
Emergency Bleeding Control Interventions After Immediate Total-Body CT Scans in Trauma Patients
}

\author{
Kaij Treskes $^{1} \cdot$ Teun P. Saltzherr ${ }^{2} \cdot$ Michael J. R. Edwards ${ }^{3} \cdot$ Benn J. A. Beuker $^{4} \cdot$ D. Den \\ Hartog $^{5} \cdot$ Joachim Hohmann $^{6} \cdot$ Jan S. Luitse $^{1} \cdot$ Ludo F. M. Beenen $^{7} \cdot$ \\ Markus W. Hollmann ${ }^{8} \cdot$ Marcel G. W. Dijkgraaf ${ }^{9} \cdot$ J. Carel Goslings ${ }^{1,10}$ on behalf of the \\ REACT-2 study group
}

Published online: 16 October 2018

(C) The Author(s) 2018

\begin{abstract}
Background Immediate total-body CT (iTBCT) is often used for screening of potential severely injured patients. Patients requiring emergency bleeding control interventions benefit from fast and optimal trauma screening. The aim of this study was to assess whether an initial trauma assessment with iTBCT is associated with lower mortality in patients requiring emergency bleeding control interventions.

Methods In the REACT-2 trial, patients who sustained major trauma were randomized for iTBCT or for conventional imaging and selective CT scanning (standard workup; STWU) in five trauma centers. Patients who underwent emergency bleeding control interventions following their initial trauma assessment with iTBCT were compared for mortality and clinically relevant time intervals to patients that underwent the initial trauma assessment with the STWU.

Results In the REACT-2 trial, 1083 patients were enrolled of which $172(15.9 \%)$ underwent emergency bleeding control interventions following their initial trauma assessment. Within these 172 patients, $85(49.4 \%)$ underwent iTBCT as primary diagnostic modality during the initial trauma assessment. In trauma patients requiring emergency bleeding control interventions, in-hospital mortality was $12.9 \%$ (95\% CI 7.2-21.9\%) in the iTBCT group compared to $24.1 \%$ (95\% CI $16.3-34.2 \%$ ) in the STWU group $(p=0.059)$. Time to bleeding control intervention was not reduced; $82 \mathrm{~min}$ (IQR 5-121) versus $98 \mathrm{~min}$ (IQR 62-147), $p=0.108$.

Conclusions Reduction in mortality in trauma patients requiring emergency bleeding control interventions by iTBCT could not be demonstrated in this study. However, a potentially clinically relevant absolute risk reduction of $11.2 \%$ (95\% CI -0.3 to $22.7 \%$ ) in comparison with STWU was observed.

Trial registration ClinicalTrials.gov: NCT01523626.
\end{abstract}

Kaij Treskes

k.treskes@amc.nl

Teun P. Saltzherr

t.saltzherr@haaglandenmc.nl

Michael J. R. Edwards

michael.Edwards@radboudumc.nl

Benn J. A. Beuker

b.j.a.beuker@umcg.nl

D. Den Hartog

d.denhartog@erasmusmc.nl

Joachim Hohmann

j.hohmann@web.de
Jan S. Luitse

j.s.luitse@amc.uva.nl

Ludo F. M. Beenen

1.f.beenen@amc.uva.nl

Markus W. Hollmann

m.w.hollmann@amc.uva.nl

Marcel G. W. Dijkgraaf

m.g.dijkgraaf@amc.uva.nl

J. Carel Goslings

j.c.goslings@olvg.nl 


\section{Background}

Improvements in speed and accuracy of computed tomography (CT) make immediate total-body CT (iTBCT) feasible as a diagnostic tool in the primary care for severe trauma patients. iTBCT scanning in trauma patients is safe, shortens the time to end of diagnostic imaging and does not increase direct medical costs [1]. However, it does not improve survival in the total group of severe trauma patients [1]. Which patients exactly could benefit from this fast and detailed diagnostic approach remains unclear.

Patients requiring emergency bleeding control interventions benefit from fast and optimal trauma screening, obtaining as much information on the bleeding site(s) as is safely possible. iTBCT during the initial trauma assessment might improve survival in this specific patient group. Time to surgery is reported to be shorter for patients requiring emergency surgery after total-body CT scanning [2]. Potential survival benefits associated with total-body CT scanning in severely injured patients requiring bleeding control measurements have been described previously [3].

The aim of this study was to assess whether an initial trauma screening with iTBCT is associated with lower inhospital mortality and shorter clinically relevant time intervals in patients requiring emergency bleeding control interventions compared to trauma screening with conventional imaging and selective CT scanning of specific body regions.

\section{Methods}

\section{Study design and patient selection}

In the REACT-2 trial, non-pregnant patients, 18 years and over, who sustained a major trauma, were included on compromised vital parameters, clinical suspicion of specific severe injuries or high-risk trauma mechanism in five trauma centers in the Netherlands and Switzerland between April 21, 2011 and January 1, 2014. Patients were considered eligible when meeting one or more of the inclusion criteria and none of the exclusion criteria shown in Table 4 of the Appendix.

Patients were randomized for iTBCT or conventional imaging with selective CT of specific body regions. Decision of eligibility by the trauma leader as well as documentation of the indication by a trauma team member was performed before the start of radiologic imaging. Potential life-saving interventions were performed prior to radiologic imaging when indicated, e.g., endotracheal intubation or chest tube placement. iTBCT was performed without preceding conventional imaging and consisted of an unenhanced CT of the head and neck and a contrast enhanced CT of thorax, abdomen and pelvis. The design of the REACT-2 study has been previously described (ClinicalTrials.gov: NCT01523626) and published [1, 4]. The REACT-2 study was approved by the medical research ethics committees at all participating centers (AMC MEC 10/145).

For this study, patients who underwent emergency bleeding control interventions following their initial trauma assessment were selected for further analysis. Emergency bleeding control interventions were defined as thoracotomy, laparotomy, external fixation of the pelvis or extremities and angiographic embolization. Multitrauma patients were defined by an Injury Severity Score (ISS) $\geq 16$ for an exploratory subgroup analysis. In addition to the intention-to-treat analysis, a per-protocol analysis was performed in which crossovers (i.e., patients who received the opposite intervention to which they had been allocated) were excluded.

Time intervals were prospectively recorded and started as the patient arrived in the trauma resuscitation room. Time to end of imaging was defined as the time from arrival in the trauma room to the end of imaging of the initial trauma assessment. Time to diagnosis was defined as

6 Department of Radiology and Nuclear Medicine, University of Basel Hospital, Petersgraben, 4031 Basel, Switzerland

7 Department of Radiology, Amsterdam University Medical Center, University of Amsterdam, Meibergdreef 9, 1105 AZ Amsterdam, Netherlands

8 Department of Anaesthesiology, Academic Medical Center, Meibergdreef 9, 1105 AZ Amsterdam, Netherlands

9 Clinical Research Unit, Department of Clinical Epidemiology, Biostatistics and Bioinformatics, Amsterdam University Medical Center, University of Amsterdam, Meibergdreef 9, 1105 AZ Amsterdam, Netherlands

10 Department of Surgery, Onze Lieve Vrouwe Gasthuis, Jan Tooropstraat 164, 1061 AE Amsterdam, Netherlands 
the time from arrival to the time all life-threatening injuries were diagnosed according to the trauma team leader. Time at the ED (emergency department) was defined by the time of arrival to the time of departure from the trauma room. Time to intervention was defined by the time of arrival to the time an emergency bleeding control intervention was initiated. Hypotension was defined as systolic blood pressure below $90 \mathrm{mmHg}$.

\section{Statistical analysis}

Continuous data with a normal distribution are presented as means and standard deviations. The non-normally distributed data are presented as medians with interquartile range. Independent sample $t$ tests and Mann-Whitney $U$ tests were used to compare the parametric and nonparametric continuous data, respectively. The Chi-squared test or Fisher's exact test was used to compare the categorical variables. The $95 \%$ confidence intervals for proportions were calculated with the modified Wald method. A $p$ value of less than 0.05 was considered statistically significant. All statistical analyses were performed with SPSS version 24 (SPSS inc., Chicago, Illinois).

\section{Results}

In the REACT-2 trial, 1083 patients were enrolled of which 172 (15.9\%) underwent emergency bleeding control interventions directly following their initial trauma assessment. Within these 172 patients, 85 (49.4\%) underwent iTBCT as primary diagnostic modality. Median ISS was 27 (IQR 20-41) in the iTBCT group compared to 29 (IQR 18-41) in the standard workup (STWU) group $(p=0.994)$. Hypotension at admission was present in $21.7 \%$ of the iTBCT group compared to $20.0 \%$ in the STWU group $(p=0.788)$. Baseline demographic and clinical characteristics are presented in Table 1.

In 85 patients in the iTBCT group, 108 emergency bleeding control interventions were performed. In the STWU group, 109 emergency bleeding control interventions were performed in 87 patients. In the iTBCT group, more patients underwent external fixations of the extremities than in the STWU group (56.5 vs. $40.2 \%, p=0.033$ ). Injury severity parameters and surgical characteristics are presented in Table 2.

In-hospital mortality was $12.9 \%$ (95\% CI $7.2-21.9 \%$ ) in the iTBCT group compared to $24.1 \% \quad(95 \%$ CI $16.3-34.2 \%$ ) in the STWU group (absolute risk reduction: $11.2 \%, 95 \%$ CI -0.3 to $22.7 \% ; p=0.059$ ). Time to diagnosis was reduced for patients who underwent iTBCT: $45 \mathrm{~min}$ (IQR 35-60) versus $57 \mathrm{~min}$ (IQR 43-85), $p=0.009$. Time to bleeding control intervention was not
Table 1 Demographic and clinical characteristics*

\begin{tabular}{|c|c|c|}
\hline Characteristic & $\begin{array}{l}\text { Total-body } \\
\text { CT }(n=85)\end{array}$ & $\begin{array}{l}\text { Standard } \\
\text { workup }(n=87)\end{array}$ \\
\hline Age $(\text { years })^{a}$ & $41(26-56)$ & $46(28-60)$ \\
\hline Male sex, $n(\%)$ & $69(81.2)$ & $66(75.9)$ \\
\hline Blunt trauma, $n(\%)$ & $82(96.5)$ & $85(97.7)$ \\
\hline \multicolumn{3}{|l|}{ Comorbidity, $n(\%)$} \\
\hline ASA I or II & $78(96.3)$ & $79(97.5)$ \\
\hline ASA III, IV or V & $3(3.7)$ & $2(2.5)$ \\
\hline \multicolumn{3}{|l|}{ In-hospital vital parameters } \\
\hline $\begin{array}{l}\text { Respiratory rate } \\
\quad(\text { per minute })^{\mathrm{a}}\end{array}$ & $16(14-20)$ & $16(14-20)$ \\
\hline Pulse $(\mathrm{bpm})^{\mathrm{b}}$ & $99(20)$ & $95(26)$ \\
\hline $\begin{array}{l}\text { Systolic blood pressure } \\
(\mathrm{mmHg})^{\mathrm{b}}\end{array}$ & $117(28)$ & $115(28)$ \\
\hline GCS (points) ${ }^{\mathrm{a}}$ & $11(3-15)$ & $11(3-15)$ \\
\hline Revised Trauma Score ${ }^{a}$ & $7.11(4.09-7.84)$ & $6.90(4.09-7.84)$ \\
\hline
\end{tabular}

ASA American Society of Anaesthesiologists

$* p>0.05$ for all between-group comparisons

${ }^{\mathrm{a}}$ Median (interquartile range)

${ }^{\mathrm{b}}$ Mean (SD)

Table 2 Injury severity and surgical characteristics*

\begin{tabular}{lll}
\hline Characteristic & $\begin{array}{l}\text { Total-body } \\
\text { CT }(n=85)\end{array}$ & $\begin{array}{l}\text { Standard } \\
\text { workup }(n=87)\end{array}$ \\
\hline Abbreviated Injury Scale $\geq 3, n(\%)$ & \\
Head & $37(43.5)$ & $32(36.8)$ \\
Chest & $52(61.2)$ & $51(49.5)$ \\
Abdomen & $27(31.8)$ & $38(43.7)$ \\
Extremities & $62(72.9)$ & $57(65.5)$ \\
Emergency interventions, & 108 & 109 \\
$n(\%)$ & & \\
Thoracotomy & $7(8.2)$ & $6(6.9)$ \\
Laparotomy & $20(23.5)$ & $32(36.8)$ \\
External fixation of the pelvis & $19(22.4)$ & $19(21.8)$ \\
External fixation of & $48(56.5)$ & $35(40.2)$ \\
$\quad$ extremities & & $17(19.5)$ \\
Angiographic embolization & $14(16.5)$ & $29(18-41)$ \\
Injury Severity Score (points) & $27(20-41)$ & $72(82.8)$ \\
Multitrauma patients, $n(\%)^{\mathrm{a}}$ & $75(88.2)$ & $24(27.6)$ \\
TBI patients, $n(\%)^{\mathrm{a}}$ & $29(34.1)$ & $0.89(0.48-0.98)$ \\
TRISS, survival probability & $0.84(0.30-0.97)$ &
\end{tabular}

Data are number (\%) or median (interquartile range)

TRISS Trauma and Injury Severity Score

$* p>0.05$ for all between-group comparisons except for external fixation of extremities $(p=0.033)$

${ }^{a}$ Multitrauma patients are defined as ISS $\geq 16$. Traumatic brain injury (TBI) patients are defined as GCS $<9$ at presentation and AIS Head $\geq 3$ 
Table 3 Outcome for patients requiring emergency bleeding control interventions

\begin{tabular}{lll}
\hline Characteristic & Total-body CT $(n=85)$ & Standard workup $(n=87)$ \\
\hline Mortality; $n, \%(95 \%$ CI $)$ & & $n=21$ \\
In-hospital mortality & $n=11$ & $24.1 \%(16.3-34.2)$ \\
& $12.9 \%(7.2-21.9)$ & $n=6$ \\
24-h mortality & $n=4$ & $6.9 \%(2.9-14.5)$ \\
& $4.7 \%(1.5-11.9)$ & \\
Time intervals, minutes (IQR) & $30(18-42)$ & $38(28-56)$ \\
Time to end of imaging & $45(35-60)$ & $57(43-85)$ \\
Time to diagnosis & $59(44-94)$ & $79(57-105)$ \\
Time at ED & $82(57-121)$ & $98(62-147)$ \\
Time to intervention & $n=39$ & $n=42$ \\
Complications; $n$, \% (95\% CI) & $45.9 \%(35.7-56.4)$ & $48.3 \%(38.1-58.6)$ \\
& & \\
Length of stay, days (IQR) & $23(12-37)$ & $20(10-33)$ \\
Total hospital stay & $5(2-12)$ & $6(2-12)$ \\
ICU stay & $3(1-9)$ & $3(1-8)$ \\
Ventilation days & $0.059^{\dagger}$
\end{tabular}

Data are number, \% (95\% confidence interval by modified Wald) or median (interquartile range)

$O R$ odds ratio, $C I$ confidence interval, $E D$ emergency department

*Chi-squared test, ${ }^{\dagger}$ Fisher's exact test, ${ }^{*}$ Mann-Whitney $U$ test

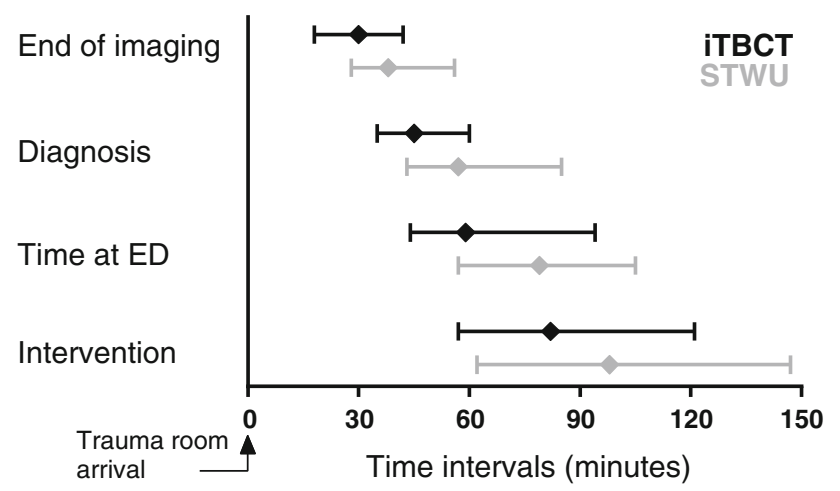

Fig. 1 Clinically relevant time intervals. Medians and interquartile ranges of clinically relevant time intervals (minutes) are displayed per randomisation group. $p<0.05$ for time to end of imaging, time to diagnosis and time at ED. iTBCT immediate total-body CT, STWU standard workup, $E D$ emergency department

reduced: iTBCT $82 \mathrm{~min}$ (IQR 57-121) versus STWU $98 \mathrm{~min}$ (IQR 62-147), $p=0.108$. Outcomes for patients requiring emergency bleeding control interventions are presented in Table 3 and time intervals are displayed in Fig. 1.

In an exploratory analysis in the group of multitrauma patients, in-hospital mortality was reduced after iTBCT compared to the STWU group: $13.3 \%$ (95\% CI 7.2-23.0\%) versus $27.8 \%$ (95\% CI $18.7-39.1 \%$ ), with an absolute risk reduction of $14.4 \%$ (95\% CI $1.6-27.3 \%, p=0.030)$. Time to diagnosis was reduced for patients who underwent
iTBCT: $47 \mathrm{~min}$ (IQR 35-61) versus $57 \mathrm{~min}$ (IQR 42-83), $p=0.033$. Time to bleeding control intervention was not reduced: iTBCT $78 \mathrm{~min}$ (IQR 56-120) versus STWU 92 min (IQR 62-125), $p=0.306$. Outcomes for multitrauma patients (ISS $\geq 16$ ) requiring emergency bleeding control interventions are presented in Table 5 of the Appendix.

In the per-protocol analysis, two crossovers were excluded. No relevant differences in outcome were found for all endpoints in comparison with the original intentionto-treat analysis as shown in Table 6 of the Appendix. With multivariate analyses on in-hospital mortality corrected for center and type of intervention and analyses on time to intervention stratified for center and type of intervention, no relevant differences were found in comparison with the original analyses.

\section{Discussion}

This study could not demonstrate a beneficial effect on survival of iTBCT for trauma patients requiring emergency bleeding control interventions. However, a potentially clinically relevant absolute risk reduction of $11.2 \%(95 \%$ CI -0.3 to $22.7 \%$ ) in comparison with STWU was observed. The original study had been powered to detect an absolute risk reduction of 5\% (from 12 to $7 \%$ ) in severe trauma patients, irrespective of their need for emergency 
bleeding control interventions, but was underpowered for the analysis in the subgroup requiring such intervention.

The potential reduction in mortality by iTBCT after major trauma could be the effect of a faster trauma workup. In addition, the complete information by iTBCT before treatment could sharpen the indication of the intervention and help the team to prepare and prioritize in the case of multiple targets for interventions. This hypothesis is supported by the study from Wada et al. [3] who reported reduced mortality for patients receiving TBCT before emergency bleeding control measurements in a retrospective study in two trauma centers. In contrast to Wada et al. [3], and Wurmb et al. [2] report unchanged mortality by TBCT for patients requiring any surgery immediately after resuscitation in multiple trauma patients in a retrospective single-center study. However, they concluded that an improvement in outcome might be assumed since the patients receiving TBCT were more severely injured [2]. This difference in injury severity could be explained by the use of a triage scheme for the TBCT group, selecting more severely injured patients for TBCT.

Huber-Wagner et al. [5] report reduced mortality for trauma patients in moderate and severe shock that underwent TBCT in a large retrospective multicenter study. Ordonez et al. report no mortality reduction in hemodynamically unstable trauma patients after CT, however, did report a survival benefit for hemodynamically unstable patients with an ISS $\geq 25$ in a single-center retrospective study. Furthermore, they report changes in indication and planning for surgery in a substantial part of the patients [6]. This further supports the use of TBCT for severely injured bleeding patients requiring fast treatment.

The relationship between iTBCT and mortality could be further supported if we could demonstrate not only a reduction in time to diagnosis but also a reduction in time to intervention. Several studies did find a benefit for time to intervention after TBCT in retrospective studies $[2,3,7]$. In the present study, there was a wide range of time to intervention intervals which could be the effect of potential confounders as center of treatment and/or different intervention types. Analyses on time to intervention stratified for center and type of intervention did not show differences compared to the original analyses.

The decision to perform an iTBCT is based on information obtained during the pre-hospital phase and during the in-hospital primary survey. Criteria for TBCT in trauma patients are diverse [8], and often the imaging itself is needed for the identification of a severely injured patient with the necessity for emergency bleeding control interventions. Selecting the appropriate patients for iTBCT and minimizing radiation exposure for the less severely injured patients remain a challenge.
A limitation of our study is that this subgroup analysis was unplanned at the design stage, resulting in a lack of statistical power for the detection of the observed clinically relevant contrast between the mortality rates. During the enrollment of our trial, associations between TBCT and emergency bleeding control interventions were reported and made this subgroup of specific interest and therefore legitimize the additional analysis on these patients. Strength of this multicenter study is the assessment of a prospectively enrolled and randomized population. Further research should be performed to confirm the suggested reduction in mortality by iTBCT in trauma patients requiring bleeding control interventions. Furthermore, future research should focus on how to select patients who could benefit from iTBCT after trauma.

\section{Conclusion}

This study could not demonstrate a beneficial effect on survival by the fast and detailed diagnostic workup by immediate total-body $\mathrm{CT}$ for trauma patients requiring emergency bleeding control interventions. There is probably a lack of statistical power for the detection of the potentially clinically relevant risk reduction in mortality by iTBCT. Further research should be performed to confirm the suggested reduction in mortality by iTBCT in trauma patients requiring bleeding control interventions.

Collaborators JC Sierink, NWL Schep, RW Peters, TJ Tromp, M Brink, R van Vugt, JS Harbers, MWJLA Wertenbroek, K ten Duis, PPM Rood, PP De Rooij, EMM Van Lieshout, R Bingisser, N Bless, C Zaehringer.

Funding ZonMw, the Netherlands Organisation for Health Research and Development (Grant No. 171102023).

\section{Compliance with ethical standards}

\section{Conflict of interest None.}

Open Access This article is distributed under the terms of the Creative Commons Attribution 4.0 International License (http://crea tivecommons.org/licenses/by/4.0/), which permits unrestricted use, distribution, and reproduction in any medium, provided you give appropriate credit to the original author(s) and the source, provide a link to the Creative Commons license, and indicate if changes were made.

\section{Appendix}

See Tables 4, 5 and 6. 
Table 4 Criteria for immediate total-body $\mathrm{CT}$ in trauma patients used in REACT-2 trial

Trauma patients with one of the following parameters at hospital arrival:

- respiratory rate $\geq 30 / \mathrm{min}$ or $\leq 10 / \mathrm{min}$

- pulse $\geq 120 / \mathrm{min}$

- systolic blood pressure $\leq 100 \mathrm{mmHg}$

- estimated exterior blood loss $\geq 500 \mathrm{ml}$

- Glasgow coma score $\leq 13$

OR

Patients with a clinical suspicion of one of the following diagnoses:

- fractures from at least two long bones

- flail chest, open chest or multiple rib fractures

- severe abdominal injury

- pelvic fracture

- unstable vertebral fractures/spinal cord compression

$O R$

Patients with one of the following injury mechanisms:

- fall from a height ( $>3 \mathrm{~m} />10$ feet)

- ejection from a vehicle

- death of occupant in same vehicle

- severely injured patient in same vehicle

- wedged or trapped chest/abdomen

Contra-indications

Trauma patients with one of the following characteristics were excluded:

- known age $<18$ years

- known pregnancy

- referred from another hospital

- clearly low-energy trauma with blunt injury mechanism

- any patient with a stab wound in one body region

- any patient who is judged to be too unstable to undergo a CT scan and requires (cardiopulmonary) resuscitation or immediate operation because death is imminent

Table 5 Outcome for multitrauma patients (ISS $\geq 16$ ) requiring emergency bleeding control interventions

\begin{tabular}{lcll}
\hline Characteristic & $\begin{array}{l}\text { Total-body } \\
\text { CT }(n=75)\end{array}$ & $\begin{array}{l}\text { Standard } \\
\text { workup } \\
(n=72)\end{array}$ & $p$ value \\
\hline $\begin{array}{l}\text { Mortality; } n, \%(95 \% \mathrm{CI}) \\
\text { In-hospital mortality }\end{array}$ & $n=10$ & $n=20$ & $0.030^{*}$ \\
& $13.3 \%$ & $27.8 \%$ & \\
& $(7.2-23.0)$ & $(18.7-39.1)$ & \\
24-h mortality & $n=3$ & $n=6$ & $0.320^{\dagger}$ \\
& $4.0 \%$ & $8.3 \%$ & \\
& $(0.9-11.6)$ & $(3.6-17.3)$ & \\
Time intervals, minutes (IQR) & & $0.019^{*}$ \\
Time to end of & $30(17-42)$ & $38(27-56)$ & \\
imaging & & &
\end{tabular}

Table 5 continued

\begin{tabular}{llll}
\hline Characteristic & $\begin{array}{l}\text { Total-body } \\
\text { CT }(n=75)\end{array}$ & $\begin{array}{l}\text { Standard } \\
\text { workup } \\
(n=72)\end{array}$ & $p$ value \\
\hline Time to diagnosis & $47(35-61)$ & $57(42-83)$ & $0.033^{\ddagger}$ \\
Time at ED & $65(45-99)$ & $79(57-107)$ & $0.139^{\ddagger}$ \\
Time to intervention & $78(56-120)$ & $92(62-125)$ & $0.306^{\ddagger}$ \\
Complications; $n, \%$ & $n=38$ & $n=38$ & $0.798^{*}$ \\
$\quad(95 \%$ CI) & $50.7 \%$ & $52.8 \%$ & \\
\multicolumn{1}{l}{$(39.6-61.7)$} & $(41.4-63.9)$ & \\
Length of stay, days (IQR) & $23(12-40)$ & $21(10-35)$ & $0.640^{\ddagger}$ \\
Total hospital stay & $6(2-14)$ & $6(2-14)$ & $0.910^{\ddagger}$ \\
ICU stay & $4(1-9)$ & $4(1-8)$ & $0.968^{\ddagger}$ \\
Ventilation days & & & \\
\hline
\end{tabular}

Data are number, \% (95\% confidence interval by modified Wald) or median (interquartile range)

$O R$ odds ratio, $C I$ confidence interval, ISS Injury Severity Score, ED emergency department

*Chi-squared test; ${ }^{\dagger}$ Fisher's exact test; ${ }^{*}$ Mann-Whitney $U$ test

Table 6 Outcome by per-protocol analysis for patients requiring emergency bleeding control interventions

\begin{tabular}{|c|c|c|c|}
\hline Characteristic & $\begin{array}{l}\text { Total-body } \\
\text { CT }(n=84)\end{array}$ & $\begin{array}{l}\text { Standard } \\
\text { workup } \\
(n=86)\end{array}$ & $p$ value \\
\hline \multicolumn{4}{|c|}{ Mortality; $n, \%$ (95\% CI) } \\
\hline \multirow[t]{2}{*}{ In-hospital mortality } & $n=11$ & $n=21$ & $0.059^{*}$ \\
\hline & $\begin{array}{l}13.1 \% \\
\quad(7.3-22.1)\end{array}$ & $\begin{array}{l}24.4 \% \\
\quad(16.5-34.5)\end{array}$ & \\
\hline \multirow[t]{2}{*}{ 24-h mortality } & $n=4$ & $n=6$ & $0.747^{\dagger}$ \\
\hline & $\begin{array}{l}4.8 \% \\
\quad(1.5-12.0)\end{array}$ & $\begin{array}{l}7.0 \% \\
\quad(3.0-14.7)\end{array}$ & \\
\hline \multicolumn{4}{|c|}{ Time intervals, minutes (IQR) } \\
\hline $\begin{array}{l}\text { Time to end of } \\
\text { imaging }\end{array}$ & $30(18-42)$ & $38(28-57)$ & $0.008^{*}$ \\
\hline Time to diagnosis & $45(35-60)$ & $57(43-85)$ & $0.008^{*}$ \\
\hline Time at ED & 59 (44-94) & $82(57-105)$ & $0.033^{*}$ \\
\hline Time to intervention & $82(62-121)$ & $96(62-135)$ & $0.230^{*}$ \\
\hline \multirow{2}{*}{$\begin{array}{l}\text { Complications; } n, \% \\
\quad(95 \% \text { CI) }\end{array}$} & $n=39$ & $n=42$ & $0.753^{*}$ \\
\hline & $\begin{array}{l}46.4 \% \\
\quad(36.2-57.0)\end{array}$ & $\begin{array}{l}48.8 \% \\
\quad(38.6-59.2)\end{array}$ & \\
\hline \multicolumn{4}{|c|}{ Length of stay, days (IQR) } \\
\hline Total hospital stay & $23(12-37)$ & $21(10-33)$ & $0.612^{*}$ \\
\hline ICU stay & $6(2-12)$ & $6(2-13)$ & $0.861^{*}$ \\
\hline Ventilation days & $3(1-9)$ & $4(1-8)$ & $0.939^{*}$ \\
\hline
\end{tabular}

Data are number, \% (95\% confidence interval by modified Wald) or median (interquartile range)

$C I$ confidence interval, $E D$ emergency department

*Chi-squared test; ${ }^{\dagger}$ Fisher's exact test; ${ }^{*}$ Mann-Whitney $U$ test 


\section{References}

1. Sierink JC, Treskes K, Edwards MJ et al (2016) Immediate totalbody CT scanning versus conventional imaging and selective CT scanning in patients with severe trauma (REACT-2): a randomised controlled trial. Lancet 388:673-683

2. Wurmb TE, Quaisser C, Balling H et al (2011) Whole-body multislice computed tomography (MSCT) improves trauma care in patients requiring surgery after multiple trauma. Emerg Med J 28:300-304

3. Wada D, Nakamori Y, Yamakawa K et al (2013) Impact on survival of whole-body computed tomography before emergency bleeding control in patients with severe blunt trauma. Crit Care 17:R178

4. Sierink JC, Saltzherr TP, Beenen LF et al (2012) A multicenter, randomized controlled trial of immediate total-body CT scanning in trauma patients (REACT-2). BMC Emerg Med 12:4
5. Huber-Wagner S, Biberthaler P, Haberle S et al (2013) Wholebody $\mathrm{CT}$ in haemodynamically unstable severely injured patients - a retrospective, multicentre study. PLoS ONE 8:e68880

6. Ordonez CA, Herrera-Escobar JP, Parra MW et al (2016) Computed tomography in hemodynamically unstable severely injured blunt and penetrating trauma patients. J Trauma Acute Care Surg 80:597-602. https://doi.org/10.1097/TA.00000000000 00975 (discussion 602-593)

7. Weninger P, Mauritz W, Fridrich P et al (2007) Emergency room management of patients with blunt major trauma: evaluation of the multislice computed tomography protocol exemplified by an urban trauma center. J Trauma 62:584-591. https://doi.org/10.1097/01. ta.0000221797.46249.ee

8. Treskes K, Saltzherr TP, Luitse JS et al (2017) Indications for total-body computed tomography in blunt trauma patients: a systematic review. Eur J Trauma Emerg Surg 43:35-42 